\title{
The Visual Hull of Piecewise Smooth Objects
}

\author{
Andrea Bottino, Aldo Laurentini \\ Dipartimenti di Automatica e Informatica, Politecnico di Torino \\ andrea.bottino@polito.it, aldo.laurentini@polito.it
}

\begin{abstract}
The visual hull relates the shape of an object to its silhouettes. This paper develops the theory of the visual hull of piecewise smooth objects, as those used in CAD applications. We show that the surface of the visual hull can be constructed using patches of nine visual event surfaces of the aspect graph of the object. A detailed analysis allows to prune away many surfaces and patches that are not relevant to the construction. Examples of construction of visual hulls are presented.
\end{abstract}

\section{Introduction}

Many computer vision algorithms are based on particular image lines. The lines called occluding contours or limbs are the projection of the contour generators, which are loci of points where there is a depth discontinuity along the line of sight.

Silhouette based recognition and reconstruction techniques use the contours that occlude the background. Volume Intersection (VI) reconstructs 3D shapes from multiple silhouettes [4], [14]. The contours occluding the background are also used for extracting shape and orientation of rotating objects [15], [25]. The geometric concept of visual hull [11] puts understanding 3D shapes from silhouettes on a firm theoretical ground.

The aspect graph, a user-centered object representation first proposed by Koenderink and van Doorn [9], takes into account the contours that occlude both the background and the object itself, and the image lines, which are projections of the creases (surface normal discontinuities).

This paper develops the theory of the visual hull of piecewise smooth surfaces, including planar surfaces, such as those used in CAD applications. The paper links visual hull and aspect graph of these objects, and extends the results presented in [1] and [13] for smooth surface objects. In the rest of the introduction we overview the basic concepts of visual hull and aspect graph.

The visual hull $(V H)$ is a geometric entity that allows understanding capabilities and limits of the techniques for recognizing or reconstructing concave 3D shapes using silhouettes [11]. Let a visual line or free line relative to an object $\mathbf{O}$ be straight line not sharing any point with $\mathbf{O}$. The visual hull $\mathbf{V H}(\mathbf{O})$ is defined as follows: $a$ point $\mathbf{p}$ belongs to $\mathbf{V H}(\mathbf{O})$ iff no free line relative to $\mathbf{O}$ passes through $\mathbf{p}$. The $\mathbf{V H}(\mathbf{O})$ is the largest object silhouette-equivalent to $\mathbf{O}$, i.e. that produces the same silhouettes as $\mathbf{O}$ observed from any possible viewpoints outside the convex hull $\mathbf{C H}(\mathbf{O})$ of $\mathrm{O} . \mathrm{VH}(\mathrm{O})$ is 
also the closest approximation of $\mathbf{O}$ that can be obtained by VI using viewpoints outside $\mathbf{C H}(\mathbf{O})$.

An intuitive physical construction of the VH is as follows. Fill the concavities of the object with soft material, and scrape off the excess material with a ruler grazing the hard surface of the object in all possible ways. The result is the VH. In Fig.1, $\mathbf{O}$ is one half of an object of revolution, $\mathbf{C H}(\mathbf{O})$ its convex hull, where the concavity has been filled. The last image shows $\mathbf{V H}(\mathbf{O})$, and in particular $S{ }^{\prime}{ }_{\mathrm{VH}}$, the surface that covers the concavity.
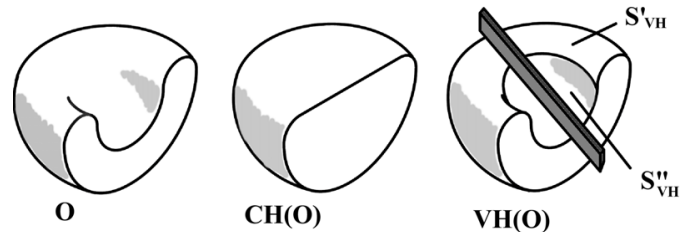

Fig.1. A piecewise smooth object $\mathbf{O}$, its convex hull $\mathbf{C H}(\mathbf{O})$ and its $\mathrm{VH}$ VH(O) Algorithms for computing the $\mathrm{VH}$ of polygons, polyhedra, solids of revolution and smooth surface objects can be found in [1], [11-13], and [17].

The basic idea of the aspect graph is the topological clustering of the line drawings of the object, consisting of occluding contours and projections of the creases. The range of all possible viewpoints can be partitioned into a set of maximal open regions where the topological structure of the line drawing, the aspect, is stable. Crossing the boundaries between these regions produces a topological change, the visual event (VE). The union of the boundaries is also called the bifurcation set. Aspects and VEs are arranged into the aspect graph (AG), where nodes are labeled with aspects, and arcs with VEs. The perspective $\mathrm{AG}$ corresponds to a $3 \mathrm{D}$ viewpoint partition, the parallel $\mathrm{AG}$ to a $2 \mathrm{D}$ partition of the Gaussian sphere.

The VEs and the related boundary surfaces (or lines for the parallel AG) depend on the object type. Planar-face objects were first considered [7]. For curved surface, the VEs can be determined as the singularities of the visual mapping [8], [16], [18], [19], [21]. Other approaches have also been used [6]. In this paper we will refer to the catalogue presented by Rieger for piecewise smooth surfaces, consisting of 19 VEs for surfaces without cross-caps [22], [23].

Algorithms for constructing the AG have been given for polyhedra, articulated objects, solids of revolution and various categories of curved objects under parallel and perspective projection [2], [5-7], [18], [20], [22-24].

The rest of the paper is organized as follows. In Section 2 we show that the VH surface consists of patches of the bifurcation set surfaces generated by nine particular VEs. In Section 3, a detailed analysis of this surface allows to prune away many surfaces and many surface patches not relevant to the construction of the VH. In Section 4 we construct the VH of two sample objects.

\section{Linking visual hull and aspect graph}

In the following we will deal with piecewise smooth algebraic surface objects, also including planar patches. We will assume that the surfaces are non singular, that there are only isolated lines making contact with the surface at more than three points of curved patches or creases, and that the curved patches are generic. Algebraic surfaces include $\mathrm{CAD}$ rational parametric patches, which can be transformed into implicit algebraic equations [10]. 
In general, the surface $\mathrm{S}_{\mathrm{VH}}$ of $\mathbf{V H}(\mathbf{O})$ can be divided in two parts: $\mathrm{S}^{\prime}{ }_{\mathrm{VH}}$ coincident with the surface $\mathrm{S}$ of $\mathbf{O}$, and $\mathrm{S}$ " ${ }_{\mathrm{vH}}$, which "covers" some concavities of $\mathbf{O}$ (Fig.1). Actually, in some cases $\mathrm{S}_{\mathrm{VH}}$ can also bound volumes not connected to $\mathbf{O}$ [11]. In this section we will relate $\mathrm{S}_{\mathrm{VH}}$ to the boundary surfaces of the $\mathrm{AG}$ of the object corresponding to nine particular VEs.

A necessary condition for a point to lie on the surface $\mathrm{S}_{\mathrm{VH}}$ of the $\mathrm{VH}$ is the following: If a point $\boldsymbol{p}$ belongs to $S_{V H}$, through $\boldsymbol{p}$ passes at least one straight line intersecting $\boldsymbol{O}$ only in boundary points.

Since the VH is the object reconstructed by VI from any possible viewpoint outside the convex hull [11], any point of $\mathrm{S}_{\mathrm{VH}}$ also belongs to the surface of (at least) a tangent cone formed by the half lines starting at a viewpoint and tangent to $\mathbf{O}$. Then, for finding points of $\mathrm{S}_{\mathrm{VH}}$ we can restrict ourselves to consider points of straight lines making contact with $\mathrm{S}$ without intersecting the object.

Clearly, if a line makes contact at only one point, this point belongs to $S^{\prime}{ }_{\mathrm{VH}}$. Then, for finding S" ${ }_{V H}$ we will consider lines making two (as in the case of Fig. 1) or three contacts at different points with $\mathrm{S}$. We have already ruled out lines making contact at more than 3 points (excluding planar faces), since these isolated lines cannot form surfaces. There are three possible contact points: crease points, fold points (where the surface is smooth) and vertices, where (for generic objects) three creases meet. Let these cases be denoted by $\mathrm{C}, \mathrm{F}$ and $\mathrm{V}$ respectively. For instance, the sequence FFC stands for contact at two fold points and at a crease point. In the following we will show that for each relevant contact case a necessary condition for a point to belong to $S{ }{ }_{\mathrm{VH}}$ is that it lies on a particular VE surface.

\subsection{Lines making three contacts}

Four cases are relevant: FFF, FFC, FCC, and CCC. We exclude cases including V, which for generic positions generate only isolated lines. In the relevant cases the link between $\mathrm{VH}$ and $\mathrm{AG}$ is immediate, since the four types of lines produce the four cases of the VE triple point (VEs 3, 9, 13, 14 of Rieger's catalogue) (Fig. 2).

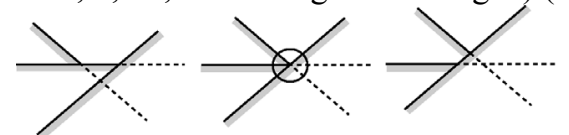

Fig.2- The VE triple point

For brevity, we will not write here the equations that determine the contact points. These can be found in [20] for parametric patches and two or three contact points, and in [18] for implicit and parametric smooth surfaces.

\subsection{Lines making two contacts}

Five cases can take place. Let us consider first the cases FF, FC, CC. Lines of these kind fill volumes, so they need a radical pruning. Let us consider a line L of any of these three types, and the orthographic projection along $\mathrm{L}$ of two infinitesimal segments of the contour generators at the contact points. It has been shown in [1] and [13] for the case FF that: a necessary condition for $L$ to contain points of $S{ }_{V H}$ is that the projections of the contour generators share a common tangent. The proof, that we omit, also holds for cases FC and CC.

This necessary condition links these cases to the AG, since the condition also specifies the three cases of the VE tangent crossing (Fig. 3), VEs 5, 10 and 12 of Rieger's catalogue. 


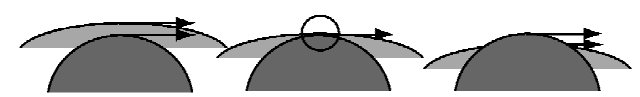

Fig. 3. The VE tangent crossing

Finally, we consider the cases VF and VC involving a vertex and a fold or crease respectively. These cases correspond directly to the surfaces generated by the two cases of the VE vertex crossing (Fig. 4), VEs 17 and 18 of Rieger's catalogue.

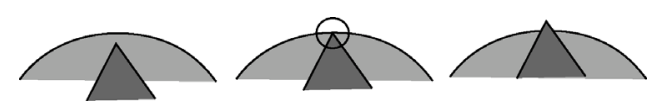

Fig. 4 -Vertex crossing

Adding up, we have shown that a necessary condition for a point to lie on $\mathrm{S}$ " ${ }_{\mathrm{VH}}$ is that it belongs to one of nine bilocal and trilocal VE surfaces.

\section{Pruning the visual event surfaces}

Constructing the VH could be done as follows.

1) Determine the surfaces generated by the nine relevant VEs

2) Construct the partition of the viewpoint space formed by these surfaces and the surface $\mathrm{S}$ of the object. Each cell of this partition belongs entirely or does not belong at all to the $\mathrm{VH}$

3) Prune the cells which do not satisfy a condition detailed in the following

4) Select and merge with the object the cells belonging to the $\mathrm{VH}$

For performing steps 1) and 2) we could exploit Rieger's algorithms for computing the AG of piecewise smooth objects [22], [23]. For pruning the cells (step 3) the following rule can be applied. Define positive the side of a relevant VE surface where free lines, compatible with the contact points, pass. A cell belonging to $\mathrm{VH}$ must lie on the negative side of all its boundary patches. Unfortunately, this condition is not always sufficient [1].

Checking if a cell belongs to VH can be done as follows. If the cell is also bounded by a patch of $\mathrm{S}$, intersect $\mathbf{O}$ with the plane tangent at a random fold point $\mathrm{P}_{\mathrm{f}}$ of this patch. If the intersection is a curve completely enclosing $\mathrm{P}_{\mathrm{f}}$, the cell belongs to VH. This is the case to face for most concavities. If the cell is not connected to $\mathbf{O}$, chose a random point in it, and construct the cone formed by the half-lines passing through the point and tangent to $\mathbf{O}$. The cell belongs to the $\mathrm{VH}$ if all the full lines containing the half lines also intersect $\mathbf{O}$ somewhere else.

However, this approach is likely to be computationally infeasible except for simple objects, considering the great number of cells $\left(\mathrm{O}\left(n^{18} d^{18}\right)\right.$ where $n$ is the number of patches and $d$ their degree [19]).

Actually, a great deal of pruning and trimming of the VE surfaces can be done before constructing the partition.

First, recall that all the VE surfaces where the generating tangent line intersects the object elsewhere must be discarded. Second, also recall that the VH cannot exceed the convex hull. Third, many more surfaces and patches can be pruned away by investigating whether free lines, compatible with the geometry of the surface at the contact points, pass through points of the VE surface. This analysis, which will be presented in the next sub-sections, extends the analysis presented in [1] and [13] for smooth objects. The lines and surfaces that do not admit free lines compatible with the contact point will be called active. 
The surfaces surviving all these pruning operations form a much restricted set of possible VH boundaries, and will be used for constructing a partition of $\mathbf{C H}(\mathbf{O})-\mathbf{O}$ containing by far less cells.

\subsection{Active surfaces making three contacts.}

Although the analysis reported in [1], [13] refers to the case of smooth surfaces, i.e. to the case FFF, it also holds without changes if one or more contours are projections of creases, that is also for cases FFC, FCC, CCC. Observe that the ruled surface making three contacts with $\mathrm{S}$ is divided by the contact curves into four patches, two unbounded external and two internal. The four contact cases are all determined by the relative spatial position of the creases (or folds) that determine the triple point. Three different spatial arrangements, shown in Fig. 5(a), (b) and (c), are possible.

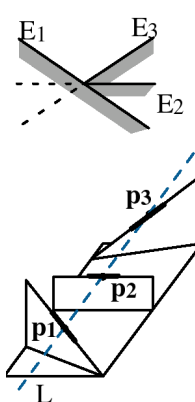

(a)
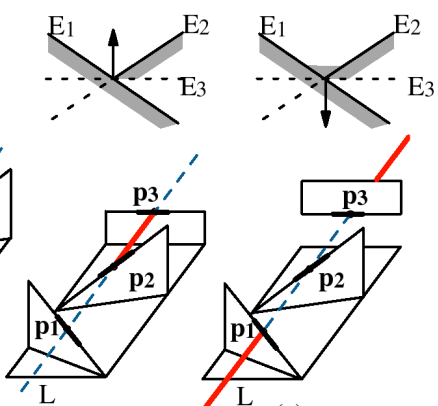

(b)

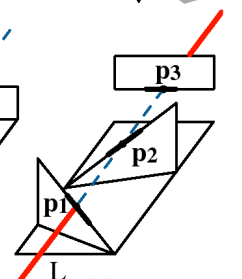

(c)

Fig.5. Active segments of lines making three contacts

For each case the figure shows the orthographic projection along $\mathrm{L}$ and a $3 \mathrm{D}$ view. In the orthographic projection the arrow, pointing outside the object, marks the limb (or crease) $\mathrm{E}_{3}$ covered by the others. In the $3 \mathrm{D}$ views only the short (actually infinitesimal) segments marked with a thicker line belong to $\mathrm{S}$. The origami-like structure supporting the segments is intended to clarify the $3 \mathrm{D}$ relative position of the contour generators. The results are summarized in Fig.5. Case (a) does not produces active surfaces; case (b) generates one internal active patch; in case (c) the two external patches are active. In the figure, the active segments are highlighted with solid lines.

\subsection{Active surfaces making two contacts.}

In this case the lines making two contacts and the surface generated are divided into one internal and two external unbounded parts. We will discuss first the cases FF, CC, CF, and afterwards the cases VF and VC.

Active surfaces FF, CC, CF

The case FF and its various sub-cases have been discussed in [1], [13] to which the reader is referred for details. The sub-cases are determined by the gaussian curvature of $\mathrm{S}$ at the tangency points. Since the gaussian curvature is not defined for creases, here we will present the arguments and results of [1], [13] in a different way, so that they directly apply to cases FF and CF.

Consider the line L, making two contacts with $\mathrm{S}$ at $\mathbf{p}_{1}$ and $\mathbf{p}_{2}$, a point $\mathbf{p}$ of this line, and an infinitesimal rotation of $\mathrm{L}$ about $\mathbf{p}$. If such rotation is possible without intersecting the object near $\mathbf{p}_{1}$ and $\mathbf{p}_{2}$, the rotated line $\mathrm{L}^{\prime}$ is a free line and the point does not belong to $S$ " ${ }_{\mathrm{VH}}$. Let $\mathrm{C}_{1}$ and $\mathrm{C}_{2}$ be infinitesimal segments of the contour generators near $\mathbf{p}_{1}$ and $\mathbf{p}_{2}$, which can be approximated with a segment of the osculating circles. Also let $\mathrm{P}_{\mathrm{C} 1}$ and 
$\mathrm{P}_{\mathrm{C} 2}$ be the osculating planes, and $\mathbf{p}_{1}$ " and $\mathbf{p}_{2}$ " be the intersections of the rotated line $\mathrm{L}$ ' with these planes. Now, let us project all these entities orthographically onto a plane P normal to L. If in this plane the projections of both $\mathbf{p}_{1}$ " and $\mathbf{p}_{2}$ " lie on the external side of the projections of the corresponding contour generators $\mathrm{C}_{1}$ and $\mathrm{C}_{2}, \mathrm{~L}$ ' is a free line. Various sub-cases occur, according to the curvatures of the projections of the contour generators and the relative positions of the external sides. In the following, if not otherwise explicitly stated, we will refer to the projections onto $\mathrm{P}$ of the various entities, which we will indicate for simplicity with the same names of their 3D counterparts.

Consider first the case where the internal sides of the contour generators lie on the same side of the (projection) of the common tangent $\mathrm{T}$. The various sub-cases possible are shown in Fig.6. The internal sides are hatched.

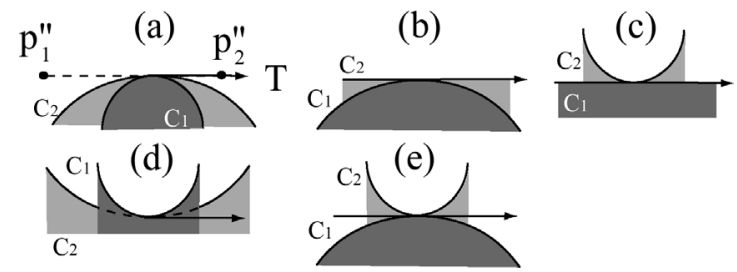

Fig.6.Sub-cases of tangent crossing where the external sides of the contour generators lie on the same side of $\mathrm{T}$

In all these sub-cases, only the internal patch is candidate to be active. Sub-case (a), where both the centers of curvature lie on the internal side, is not active, and the corresponding VE surface can be discarded. This is easily seen, since the line L can be rotated about any of its points in the plane containing $\mathrm{L}$ and $\mathrm{T}$ without intersecting the object. Similar arguments show that also sub-case (b) is not active. On the contrary, no rotation about points of the internal segment compatible with the surfaces at the contact points is possible in sub-cases (c) and (d), and therefore the internal patches are active. Sub-case (e) is more complex, and its analysis requires the values of the radii of curvature $r_{1}$ and $r_{2}$ of (the projections) of $\mathrm{C}_{1}$ and $\mathrm{C}_{2}$. It has been shown in [1], [13] that the internal segment of the $3 \mathrm{D}$ line $\mathrm{L}$ consists of an inactive segment $\mathbf{p}_{1} \mathbf{p}_{\mathrm{M}}$ starting at the point $\mathbf{p}_{1}$ of the contour generator where the center of curvature lies on the external side, and an active segment $\mathbf{p}_{\mathrm{M}} \mathbf{p}_{2}$ ending at the other point, such that

$\left|\mathbf{p}_{1} \mathbf{p}_{\mathrm{M}}\right| /\left|\mathbf{p}_{\mathrm{M}} \mathbf{p}_{2}\right|=r_{1} / r_{2}$

Consider now the sub-cases where the external sides of the contour generators lie on opposite sides of T (Fig .7).

In all these sub-cases, only the external patches are candidate to be active. It can be shown that sub-cases (a) and (b) are not active, and that sub-cases (c) and (d) generate two active external patches. The sub-case (e) requires a more complex analysis, whose results are as follows.
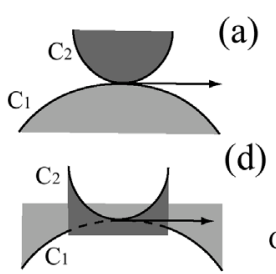

(d)

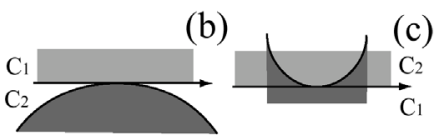

(e)

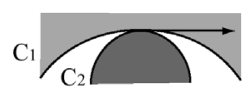

Fig.7.Sub-cases of tangent crossing where the external sides of the contour generators lie on opposite side of $\mathrm{T}$

Consider the external segment starting at $\mathbf{p}_{2}$, which is the point of the contour generator with the center of curvature on the internal side, and assume $r_{1}>r_{2}$, as in Fig.(e), The 
external segment is divided into one active part $\mathbf{p}_{2} \mathbf{p}_{\mathrm{M}}$ and an inactive part $\mathbf{p}_{\mathrm{M}} \infty$. The point $\mathbf{p}_{\mathrm{M}}$ is such that $\left|\mathbf{p}_{1} \mathbf{p}_{\mathrm{M}}\right| /\left|\mathbf{p}_{2} \mathbf{p}_{\mathrm{M}}\right|=r_{1} / r_{2}$.

If $r_{1} \leq r_{2}$, the whole external segment is active. Now consider the external segment starting at $\mathbf{p}_{1}$, and assume $r_{1} \geq r_{2}$, as in Fig. (e). In this case the external segment is totally inactive. If $r_{1}<r_{2}$, the segment $\mathbf{p}_{1} \mathbf{p}_{\mathrm{M}}$, is inactive. The remaining segment $\mathbf{p}_{\mathrm{M}}, \infty$ is active. The position of $\mathbf{p}_{\mathrm{M}^{\prime}}$ is such that $\left|\mathbf{p}_{1} \mathbf{p}_{\mathrm{M}^{\prime}}\right| /\left|\mathbf{p}_{2} \mathbf{p}_{\mathrm{M}^{\prime}}\right|=r_{1} / r_{2}$. For the case FF, for which it was originally developed in [1], [13], the above analysis only requires the type of the gaussian curvature at the tangency points, exception made for the cases (e) in Fig.6 and Fig.7, where the values of the radii of curvature of the projections of $\mathrm{C} 1$ and $\mathrm{C}_{2}$ are required. These radii can be easily obtained in 3D for a fold point (see [1], [13] for the details) and in P.

The above results also apply to the cases involving creases as contour generators, the only difference being that the contour generator equation is known a priori. From this equation we can easily find the radius of curvature in the osculating plane and in $\mathrm{P}$ (for brevity we omit the details).

\section{Active surfaces $V F$ and $V C$}

These surfaces are cones with vertex in V. Also in this case we will analyze the orthographic projections of the vertex $\mathrm{V}$ (and of two infinitesimal segments of the creases meeting at the vertex) and the contour generator $\mathrm{F}$ or $\mathrm{C}$ onto a plane normal to $\mathrm{L}$, for understanding if an infinitesimal rotation of $\mathrm{L}$ about one of its points, and compatible with the geometry of S near the contact points, is possible. Various subcases arise. The three sub-cases (a), (b) and (c) in Fig.8 all refer to a convex vertex projection.
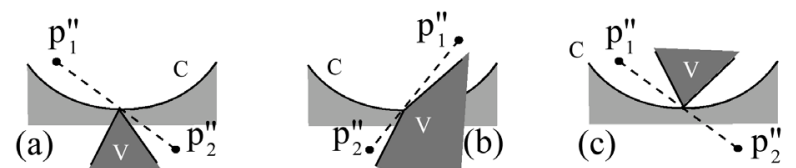

Fig.8. VC or VF sub-cases (convex vertex projection)

It is easy to see that in these sub-cases an infinitesimal rotation of $L$ about any of its points without intersecting the object near the contact points is possible, and then these surfaces can be pruned away. In the figures it is shown the trace in $\mathrm{P}$ of possible planes containing L and L'. $\mathbf{p}_{1}$ " lies on the osculating plane of the contour generator, and $\mathbf{p}_{2}$ " in the plane containing the segment of creases meeting at $\mathrm{V}$. The sign or value of the curvature of the contour generator $\mathrm{C}$ does not affect these conclusions.

The sub-cases (d), (e) and (f), shown in Fig. 9, refer to a concave vertex projection. For analyzing sub-case (d), let us consider a plane rotating about $\mathrm{L}$. Two kinds of intersections with $\mathbf{O}$ are possible. For planes as $P_{1}$ in the figure, the intersection is shown in $\left(d_{1}\right)$. It is clear that no rotation about points of the internal segment can take place in this plane. In any other plane as $P_{2}$, no rotation at all is possible $\left(\mathrm{d}_{2}\right)$. Then the internal segment is active. Sub-case (d) can be seen as a limit situation of sub-case (d) in Fig 6, for a vanishing radius of curvature of one contour generator.

Consider sub-case (e). The intersection of $\mathrm{P}_{1}$ with the object $\left(\mathrm{e}_{1}\right)$ shows that the external segments are not active. The intersection $\left(\mathrm{e}_{2}\right)$ with $\mathrm{P}_{2}$ shows that also the internal segment is not active, and then the whole surface can be pruned. Finally, consider subcase (f). Two kinds of intersections are possible. The intersection $\left(\mathrm{f}_{1}\right)$ with a plane as $\mathrm{P}_{1}$ shows that the internal segment is not active. The intersection $\left(f_{2}\right)$ with any other plane as $\mathrm{P}_{2}$ shows that the external segments are active. Sub-case (f) could be seen as a limit situation of sub-case (d) in Fig.7 
Usually, CAD objects are not generic objects. Then we will explicitly consider another case of contact at two points, since it is relevant to CAD objects. This is the case of two coplanar and rectilinear edges. It could be considered the limit situation of sub-case (d) in Fig. 6, or of sub-case (d) in Fig. 7, for vanishing curvatures of both contours. In the first case it produces a planar internal active patch, in the second two active planar external patches. Coplanar edges often generate overlapping planar active surfaces, as shown in the following examples.

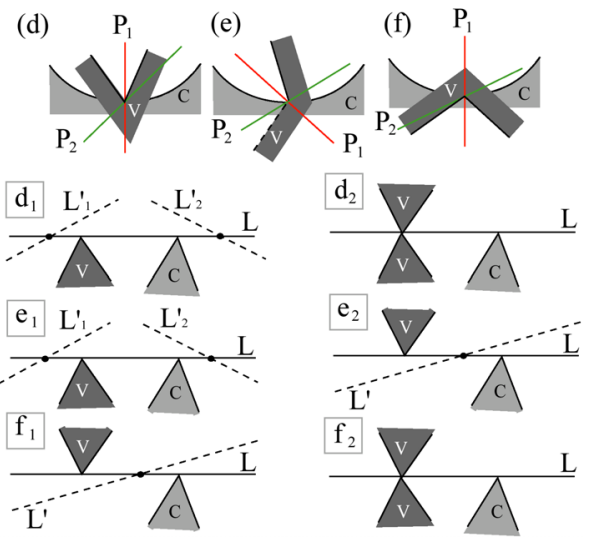

Fig. 9. VC or VF sub-cases (concave vertex projection)

\section{Examples}

For simplicity, in the examples all the active surfaces are generated by lines making two contacts.

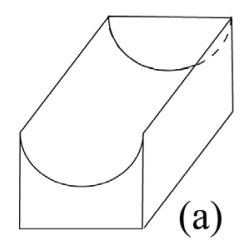

(a)

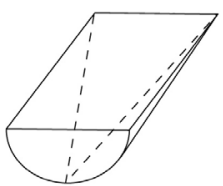

(d)

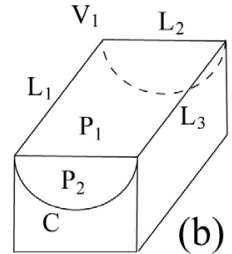

(b)

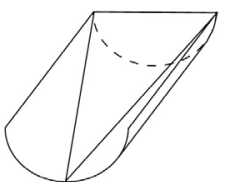

(e)
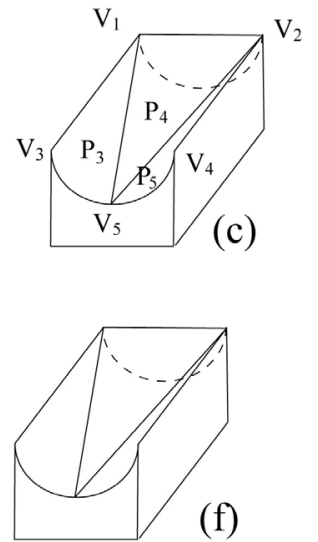

Fig.10. The object is shown in (a), the potentially boundary patches in (b) and (c), the two cells formed by these patches in (d) and (e), the VH in (f).

Example 1. Consider the object in Fig 10(a). In all, there are five potential boundary patches. Patches due to overlapping surfaces are counted once. Two planar patches, $\mathrm{P}_{1}$ and $\mathrm{P}_{2}$ are shown in Fig. 10(b). $\mathrm{P}_{1}$ is due to three patches overlapping, due to lines making contact at $\mathrm{L}_{1}$ and $\mathrm{L}_{3}, \mathrm{~L}_{1}$ and $\mathrm{L}_{2}, \mathrm{~L}_{2}$ and $\mathrm{L}_{3}$. Also, $\mathrm{P}_{2}$ is generated by various overlapping patches. In Fig. 10(c) we show three other patches, $\mathrm{P}_{3}, \mathrm{P}_{4}$, and $\mathrm{P}_{5}$. Patch $\mathrm{P}_{3}$ is generated by the lines passing through vertex $V_{1}$ and making contact at crease $C$ between $V_{3}$ and $V_{5}$. This is an instance of sub-case (d) in Fig. 9. Observe that, as soon 
as the line passing through $\mathrm{V}_{1}$ and grazing $\mathrm{C}$ becomes $\mathrm{V}_{1} \mathrm{~V}_{5}$, where $\mathrm{V}_{5}$ is the middle point of $\mathrm{C}$, the two-contact case turns into the inactive sub-case (e) in Fig. 9.

Then, all the lines passing through $V_{1}$ and making contact with $C$ between $V_{5}$ and $V_{4}$ are not active. Patch $\mathrm{P}_{4}$ is planar and is generated by the lines making contact at $\mathrm{V}_{5}$ and $\mathrm{L}_{2}$. Actually $\mathrm{V}_{5}$ is the only point of $\mathrm{C}$ that originates a tangent crossing event (sub-case (c) of Fig. 6) together with edge $\mathrm{L}_{2}$. Patch $\mathrm{P}_{5}$ is symmetric to $\mathrm{P}_{3}$.

The five patches produce a partition with two cells, shown in Fig. 10(d) and (e). The first cell does not satisfy a necessary condition to belong to the $\mathrm{VH}$, since it lies on the positive side of patches $\mathrm{P}_{3}, \mathrm{P}_{4}$, and $\mathrm{P}_{5}$. The second satisfies this condition, and it clearly belongs to the $\mathrm{VH}$, shown in Fig. 10(f).

Example 2. The object is shown in Fig.11(a). The possible boundary patches are 6 . Three planar patches, $\mathrm{P}_{1}, \mathrm{P}_{2}$ and $\mathrm{P}_{3}$ are shown in Fig. 11(b). There are 4 other planar infinite active patches $\left(\mathrm{P}_{4}, \mathrm{P}_{5}, \mathrm{P}_{6}\right.$ and $\mathrm{P}_{7}$ shown in Fig 11(b)), but they lie outside the convex hull, and can be pruned.

Lines making contact at the semi-circles $C_{1}$ and $C_{2}$ produce a conical surface whose internal part is divided into an active patch $\mathrm{P}_{8}$ and an inactive patch $\mathrm{P}_{9}$. This is an instance of sub-case (e) in Fig.6. Two other relevant patches exist, $\mathrm{P}_{10}$ and $\mathrm{P}_{11}$ in Fig $11(\mathrm{e})$ and (f), generated by the lines passing through the vertices $V_{1}$ and $V_{2}$ and crease $\mathrm{C}_{1}$. These are instances of sub-case (d) in Fig.9.

As in Example 1, the patches produce 2 connected cells (not shown for brevity in the figure). The upper cell does not lie on the negative side of all its boundary patches and then does not belong to VH. In Fig. $11(\mathrm{~g})$ we show the VH, which is bounded by $\mathrm{P}_{8}$ and by parts of $\mathrm{P}_{10}$ and $\mathrm{P}_{11}$.
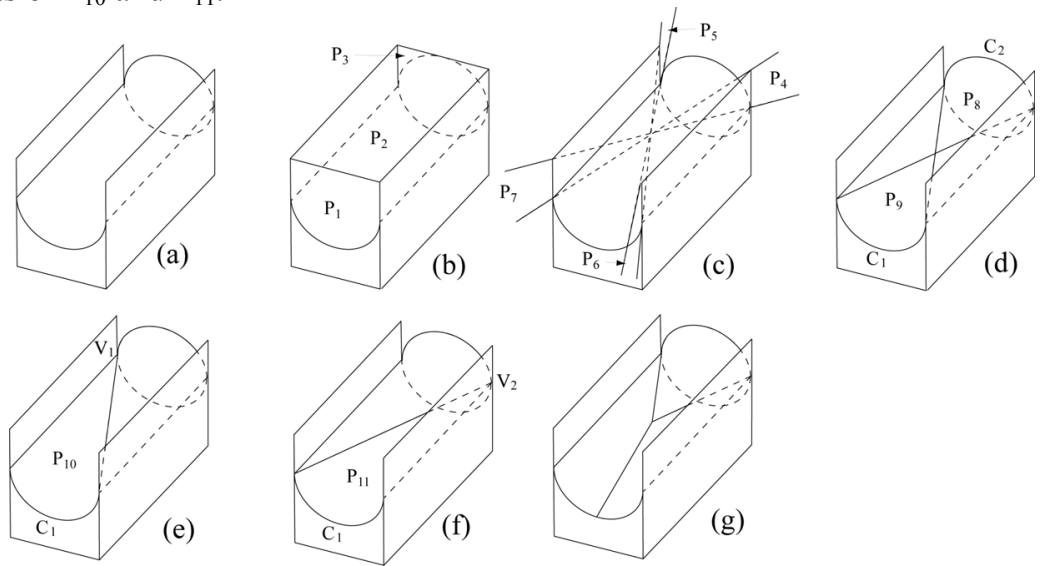

(d)

Fig.11. The object (a). Three planar potential active surfaces (b). Four planar surfaces that can be pruned (c). Only $\mathrm{P}_{8}$ is potentially active for this conical surface (d). Two symmetric potential active surfaces (e), (f). The resulting VH (g).

\section{References}

[1] A.Bottino and A.Laurentini," The visual hull of smooth curved objects," IEEE Trans. PAMI, to appear

[2] K.Bowyer , M. Sallam, D.Eggert and J. Stewman, "Computing the Generalized Aspect Graph for Objects with Moving Parts," Trans. PAMI, vol.15, no.6, pp.605610,1993

[3] J.Callahan and R. Weiss, "A Model for Describing Surface Shape," in Proc. CVPR, pp. $240-245,1985$ 
[4] C. H. Chian and J. K. Aggarwal: Model reconstruction and shape recognition from occluding contours", Trans. PAMI, Vol.11, pp.372-389, 1989

[5] D.W. Eggert, K.W. Bowyer, C.R. Dyer, H.I. Christensen, and D.B. Goldof, "The Scale Space Aspect Graph", Trans. PAMI, vol.15 (11), pp.1114-1139, 1993

[6] D.Eggert and K.Bowyer," Computing the Perspective Aspect Graph of Solids of Revolution,"Trans. PAMI, vol.15 (2), pp.109-128, 1993

[7] Z. Gigus, J. Canny, and R. Seidel, "Efficiently computing and representing aspect graphs of polyhedral objects," Trans. PAMI, vol.13 (6), pp. 542-551, 1991

[8] J. J. Koenderink and A. J. van Doorn," The Singularities of the Visual Mapping," Biol. Cybern. vol.24, pp.51-59, 1976

[9] J. J. Koenderink and A. J. van Doorn," The Internal Representation of Solid Shapes with Respect to Vision," Biol. Cybern. vol.32, pp.211-216, 1979

[10] D.J. Kriegman and J.Ponce, "On recognizing and positioning curved 3D objects from image contours," Trans. PAMI, vol.12 (12), pp.1127-1137, 1990

[11] A. Laurentini," The Visual Hull Concept for Silhouette-based Image Understanding", Trans. PAMI,vol.16, pp.150-162, 199

[12] A. Laurentini, "Computing the Visual Hull of Solids of Revolution," Pattern Recognition, vol. 32, pp.377-388, 1999

[13] A. Laurentini "The Visual Hull of Curved Objects" Proc. $7^{\text {th }}$ IEEE Int. Conf. on Comp. Vision, Kerkira, Sept. 1999

[14] W.N. Martin and J.K. Aggarwal, "Volumetric description of objects from multiple views," Trans. PAMI, vol. 5, pp.150-158, 1983

[15] P. Mendonca, K.Wong and R. Cipolla, "Epipolar geometry from profiles under circular motion," Trans. PAMI, vol.23, pp.604-616, 2001

[16] O.A. Platonova, "Singularities of projections of smooth surfaces," Russian Math. Surveys, . 39, pp.177-178, 1984

[17] S. Petitjean,"A Computational Geometric Approach to Visual Hull," Int. J. of Comput. Geometry and Appl., vol.8 (4), pp. 407-436, 1998

[18] S. Petitjean, J. Ponce and D.J. Kriegman, "Computing exact aspect graphs of curved objects: algebraic surfaces," Int. J. of Computer Vision, vol. 9, no.3, pp.231255,1992

[19] S. Petitjean," The enumerative geometry of projective algebraic surfaces and the complexity of aspect graphs," Int. J. of Computer Vision, vol. 19, pp.1-29, 1996

[20] J. Ponce and J. Kriegman, "Computing exact aspect graph of curved objects: parametric patches," Proc.AAAI Conf. Pp.1074-1079, Boston, July 1990

[21] J. Rieger, "On the classification of views of piecewise smooth objects," Image Vis. Comput. Vol.5, pp. 91-97, 1987

[22] J. Rieger," Notes on the complexity of exact view graph algorithms for piecewise smooth algebraic surfaces," Discrete \& Comput. Geometry, Vol.20, pp. 205-229, 1998

[23] J. Rieger," On the complexity and computation of view graphs of piecewise smooth algebraic surfaces," Philos. Trans. Roy. Soc. London Ser.A, pp.-1899-1940, 1996

[24] I. Shimshoni and J.Ponce, "Finite Resolution Aspect Graphs of Polyhedral Objects," IEEE Trans. Pattern Analysis and Machine Intelligence, vol.19, no.4, pp.315-327, 1997

[25] J. Y. Zheng: Acquiring 3D models from sequences of contours, Trans. PAMI, Vol. 16, no.2, pp.163-178,1994 